\title{
AN 18-KG GIANT VARIANT OF A WELL-DIFFERENTIATED RETROPERITONEAL LIPOSARCOMA OF THE KIDNEY
}

\author{
Deyan Anakievski ${ }^{1,3}$, Alexander Hinev ${ }^{1,3}$, Petar Kosev ${ }^{2,3}$, Inna Gocheva ${ }^{3}$ \\ ${ }^{1}$ Department of Surgery, Faculty of Medicine, Medical University of Varna \\ ${ }^{2}$ Department of General Medicine and Clinical Laboratory, Faculty of Medicine, \\ Medical University of Varna \\ ${ }^{3}$ Clinic of Urology, St. Marina University Hospital
}

\begin{abstract}
Well-differentiated liposarcoma (WDLS), the most common type of liposarcoma is a slow-growing, painless tumor usually located in the retroperitoneum or the limbs.

In this abstract we present a case of a 71-year-old male who presented in our clinic with a diagnosis of a giant WDLS. We performed a CT scan from which we found a giant abdominopelvic mass originating from the right kidney. This mass was pushing almost all organs and structures from the right abdominal cavity across the midline to the left side, which includes the IVC, aorta and the right ureter, all bowels were also pushed and were above this mass. A large 18-kg fibro-fatty mass was extirpated. The histopathological findings reported low-grade differentiated liposarcoma. The CT scan, which was done 3 months after the operation, determined that the patient was free from recurrence.
\end{abstract}

Keywords: retroperitoneum, liposarcoma, well-differentiated liposarcoma, giant liposarcoma

\section{INTRODUCTION}

Retroperitoneal liposarcomas are a rare subtype of soft tissue sarcomas and are divided according of their localisation and histological type $(1,2)$. About $20 \%$ of all soft tissue sarcomas are found in the retroperitoneum, and $15 \%$ of them are liposarcomas from which about one third originates from perirenal fat. The disease is usually found in middle-aged men, of-

Address for correspondence:

Deyan Anakievski, $M D$

St. Marina University Hospital

Clinic of Urology

1 Hristo Smirnenski Blvd

9010 Varna, Bulgaria

e-mail:dejan_anakievski@yahoo.com

Received: January 4, 2017

Accepted: March 20, 2017 ten without clinical manifestations and has nonspecific clinical symptoms.

\section{CASE REPORT}

The case is that of a 71-year-old man without significant medical history who presented to the urologist with a progressively distended abdomen associated with weight loss, fatigue and dyspnoea. Abdominal ultrasonography and contrast-enhanced abdominal computed tomography (CT) found a voluminous, mixed-density mass located retroperitoneally in the right perirenal area; the mass was approximately $50 \times 40 \times 50 \mathrm{~cm}$ in size, with clearly defined, irregular margins and a few visible vessels. The mass was in contact with the pancreas, the stomach (the distal third), the aorta, the liver, the gallbladder, the ascending and transverse colon, the inferior vena cava and the portal vein. The mass occupied the entire right hemiabdomen and part of the left one, 

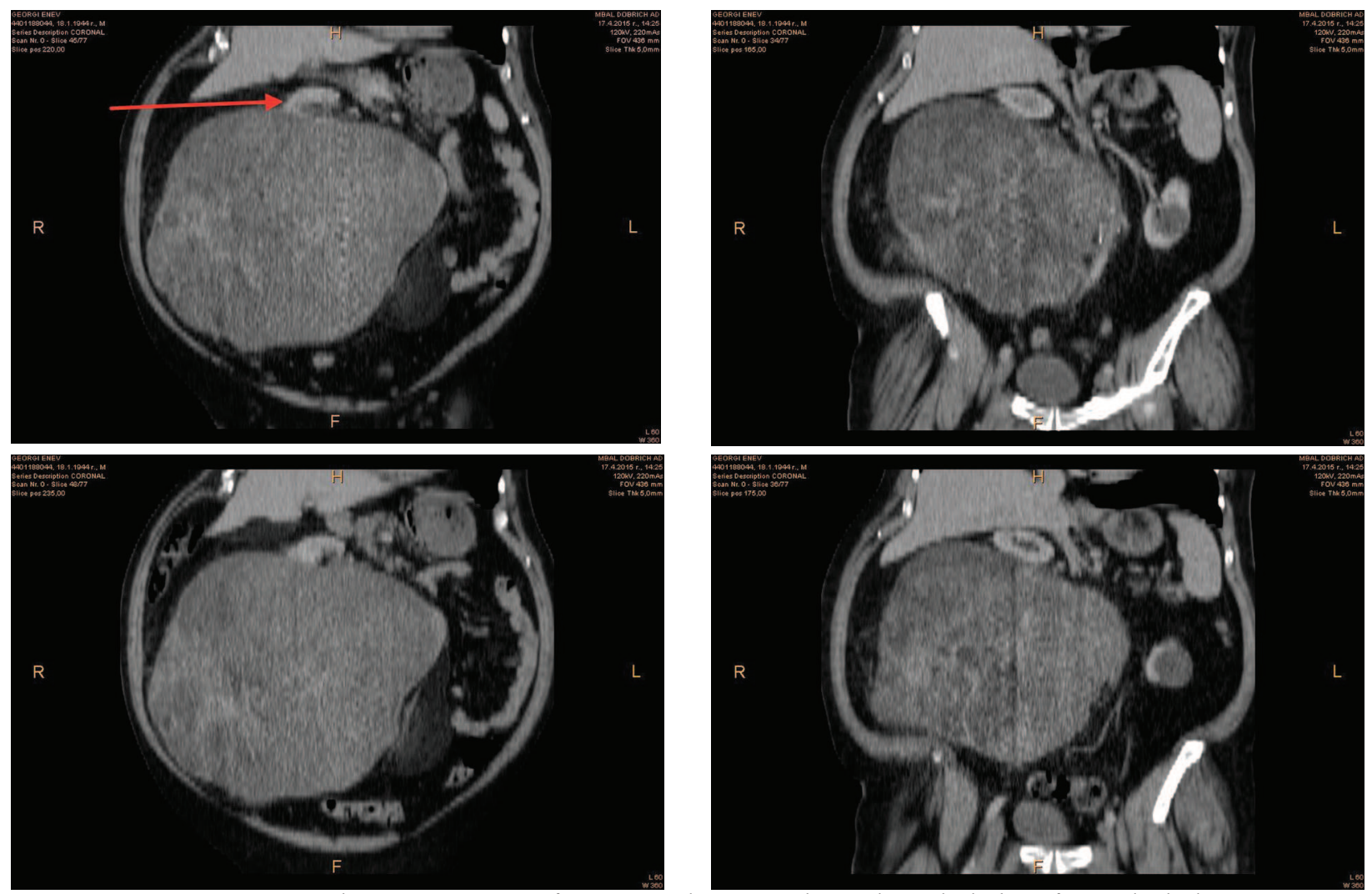

Fig. 1. Preoperative CT showing a giant Tu formation, the arrow shows the right kidney from which the tumour originates

compressing the abdominal organs, displacing the liver cranially and compressing the large vessels (Fig. $1)$.

His hemogram, renal, liver functions and CEA levels were within the normal range. After the haemodynamic status of the patient was optimised, a surgical intervention was performed via a transperitoneal approach, and a retroperitoneal mass weighing $18 \mathrm{~kg}$ was excised. Macroscopically, the tumour was lying on the posterior face of the kidney with dimensions 50x40x50 cm, and was completely capsulated by thin grey-pink capsula with peripheral fat tissue (Fig. 2). During surgery, no metastases were found in other abdominal organs, so no other organ resection was necessary.

There was no evidence of positive surgical margins. The operating time was $90 \mathrm{~min}$, without blood transfusion.

After a consultation with an oncologist, the patient did not receive adjuvant therapy. He was observed annually and with follow-ups.
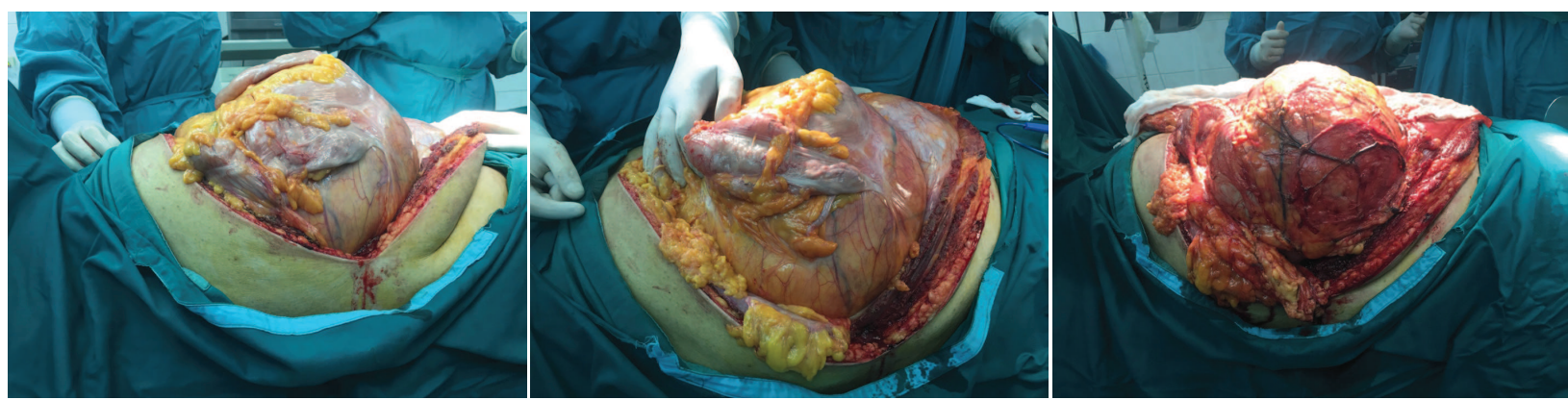

Fig. 2. Intraoperative images - all intestines are dislocated above the tumour formation 
Deyan Anakievski, Alexander Hinev, Petar Kosev et al.
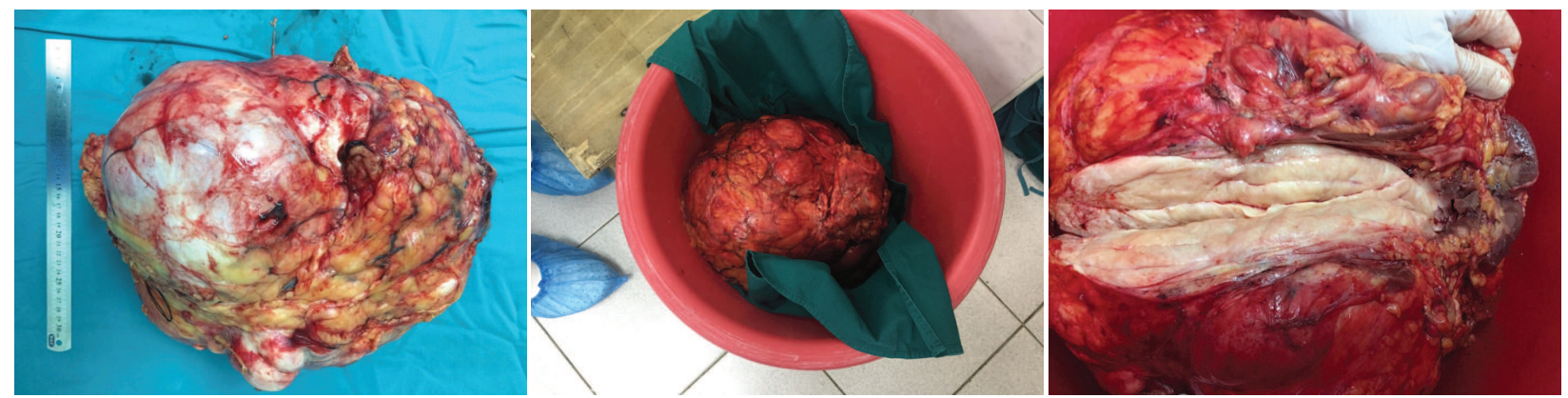

Fig. 3. Tumour formation - macroscopic view
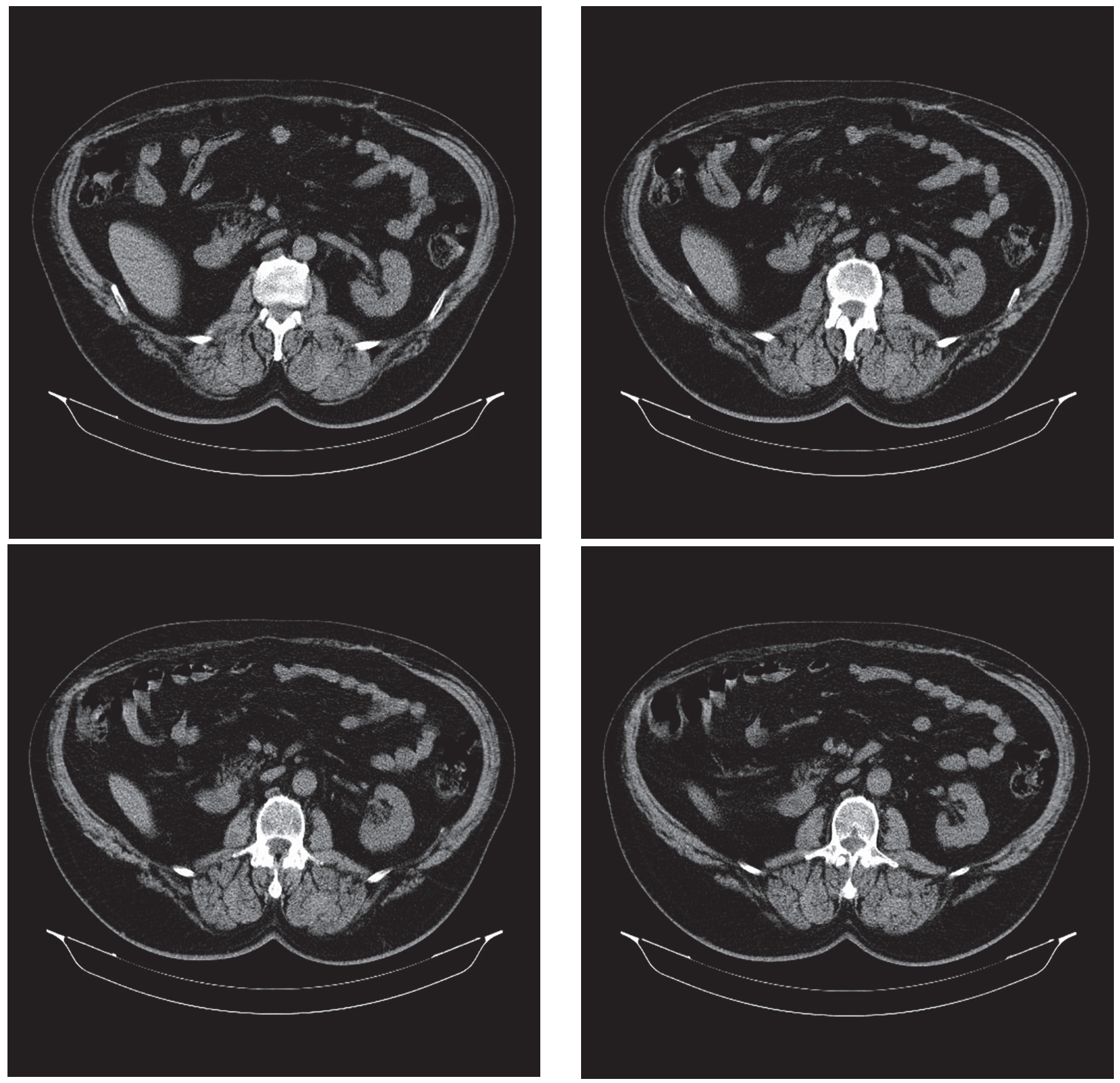

Fig. 4. CT three months later showed no MTS

Scripta Scientifica Medica, vol. 49, No. 1, 2017, pp. 53-57 


\section{DISCUSSION}

The retroperitoneum is the space in the abdominal cavity, which anatomically is behind the peritoneum. It has no specific anatomical structures and that is why tumours, either primary or metastatic, grow silently before they are clinically manifested. Retroperitoneal sarcomas are $10 \%$ to $15 \%$ of all soft tissue sarcomas. Liposarcoma is the most common malignant tumour in the retroperitoneal space. The classification of the tumour is based on the amount of lipid inside the cells, and the degree of cell differentiation. Usually these tumours are composed of mature adipocytes, which are proliferating. The incidence is $1 / 200,000$ per year, which accounts for 30 to $50 \%$ of all liposarcomas. WDLSs are slow-growing, usually painless and occur in adulthood, more often in men than in women at the age of 50 to 60 years (3). When the tumour is large, it can compress the bowels and the kidneys and symptoms like bowel obstruction, hydronephrosis, and hematuria can be observed. When a mass is found on ultrasonography, the next step is to perform computed tomography $(\mathrm{CT})$. If we find other lesions during the examination, they do not require pretreatment biopsy unless resection is likely to be incomplete or highly morbid. Surgery is the mainstay of treatment for all sarcomas, also for WDLS. If the tumour excision is complete, the patient does not need further therapy. However, this patient should be monitored for recurrence frequently by protocols. The prognosis of sarcomas depends on the tumour location and their pathological type. Liposarcomas on the extremities usually have a good prognosis after tumour removal, with very low rates of recurrence and substantially no mortality. In retroperitoneal WDLS the situation is different. However, there is a 5 -year probability of recurrence in only $54 \%$, and a 5 -year disease-specific survival is 80 to $90 \%$ (4).

Low grade or well-differentiated liposarcomas (WDLSs) are slow-growing soft tissue malignant tumours. Some genetic mutations have been observed as a cause of developing this kind of tumours. The real cause is still unknown. The radical curative treatment of these tumours is usually surgery, which includes complete excision. The prognosis of well-differentiated liposarcomas is excellent when the tumour is detected early, and because they do not metastasize.
A liposarcoma (LPS or LS) is a malignant tumour of the fat cells. It is rare and usually asymptomatic and can occur anywhere in the body.

There are 4 pathological types of liposarcomas:

a) Low grade or well-differentiated liposarcoma (WDLS): 45\%; b) Dedifferentiated liposarcoma: It is a high-grade tumour; c) Myxoid liposarcoma: 30\%; d) Pleomorphic liposarcoma (PLS): 5\% (5).

The World Health Organization (WHO) classifies well-differentiated liposarcomas into 4 types:

$\diamond$ Lipoma-like well-differentiated liposarcoma: It is with very a good prognosis if removed completely.

$\diamond$ Sclerosing well-differentiated liposarcoma: It is frequently found in the retroperitoneum and can also be in the paratesticular region. In this tumour, there is a presence of fibrosis.

$\diamond$ Inflammatory well-differentiated liposarcoma: Prominent inflammatory cells are found in the tumour.

$\diamond$ Spindle cell well-differentiated liposarcoma: In addition to the retroperitoneum and spermatic cord, it can occur in the subcutaneous tissue. There are spindle cells in the tumour, which give the tumour its name.

Incidence of well-differentiated liposarcoma is observed more frequently in men than in women. Histological examination is important when the diagnosis of liposarcomas is made, because different subtypes of sarcomas are very closely related to the prognosis of the disease. The anatomic site of the tumour is recognised as an important prognosis factor considering recent publications, which noted that patients with retroperitoneal tumours have a lower survival rate compared to those patients who have tumours in different parts of the body. Another prognostic factor is complete removal of the tumour during the surgery, which has a large impact on recurrence and survival rate (6). Also, there are publications, which report that positive surgical margins are other findings that have an impact on local recurrence, and recurrence rate is established to be from 27 to $29 \%$ in the completely excised tumours (7). Our case has had no recurrence for 3 months after the surgery and the patient is undergoing regular observation and check-ups, based on the protocol. 


\section{REFERENCES}

1. Frank RM, Velasco JM. Surgical management of incidental renal tumor during excision of retroperitoneal liposarcoma and osteogenic sarcoma. Am Surg 2013;79:E88-90.

2. Goertz RS, Lenfers BH, Goertz GH. Huge liposarcoma of the left retroperitoneum. Am J Surg 2009;197:e59-60.

3. Argani P, Facchetti F, Inghirami G, Rosai J. Lymphocyte-rich well-differentiated liposarcoma: report of nine cases. Am J Surg Pathol 1997;21:884-95.

4. Maamouri N, Cheikh I, Ouerghi H, Oukaa A, Belkahla N, Mnif E, et al . Giant retroperitoneal liposarcoma. One case report. Rev Med Interne 2005;26:145-8.

5. Henricks WH, Chu YC, Goldblum JR, Weiss SW. Dedifferentiated liposarcoma: A clinico-pathologic analysis of 160 cases. Mod Pathol 1995;8;6A.

6. Henricks WH, Chu YC, Goldblum JR, Weiss SW. Dedifferentiated liposarcoma: a clinicopathologic analysis of 155 cases with a proposal for an expanded definition of dedifferentiation. Am J Surg Pathol. 1997;21:271-81.

7. McCormick D, Mentzel T, Beham A, Fletcher CD. Dedifferentiated liposarcoma. Clinicopathologic analysis of 32 cases suggesting a better prognostic subgroup among pleomorphic sarcomas. Am J Surg Pathol. 1994;18:1213-23. 